Yujie Zhang

Chris G. Bartone

\section{A real-time meteorological-based troposphere (RMT) correction with integrity bound for long baseline DGPS}

Published online: 5 January 2006

(C) Springer-Verlag 2006

The online version of the original article can be found at http://dx.doi.org/10.1007/ s10291-005-0132-z

\section{Y. Zhang $(\bowtie)$}

Avionics Engineering Center,

Ohio University, 234H Stocker Center,

Athens, OH 45701-2979, USA

E-mail: yz315101@ohio.edu

Tel.: + 1-740-5931527

Fax: + 1-740-5931604

C. G. Bartone

School of Electrical Engineering and

Computer Science, Ohio University,

351 Stocker Center, Athens,

OH 45701-2979, USA

E-mail: bartone@ohio.edu

Tel.: + 1-740-5939573

Fax: + 1-740-5931604

\section{GPS Solut (2005).}

DOI: 10.1007/s10291-005-0132-z

The words "integrity bound" are meant to include the process of establishing an accuracy indicator (i.e., $\sigma$ ) and overbound criteria for the troposphere error estimate so that it can be monitored for integrity purposes. 